\title{
Intervenção Fisioterapêutica na Atrofia Muscular Espinhal: revisão de literatura
}

\author{
Physiotherapic intervention in spinal muscular atrophy: \\ literature review
}

\section{Intervención fisioterapéutica en la atrofia muscular espinal: revisión de la literatura}

\author{
Francielly Suzaine da Silva ${ }^{1}$, Júlia Manoela Prada Rodrigues ${ }^{2}$, \\ Rômulo Nolasco de Brito ${ }^{3}$, Thaisa Cardoso Macedo 4 , \\ Andrea Delfino Borgmann ${ }^{5}$
}

1.Curso de fisioterapia, Mestranda em Ciências da Saúde - LaNEx, Universidade do Sul de Santa Catarina, Palhoça-SC, Brasil.

2. Curso de fisioterapia, Universidade do Sul de Santa Catarina, Palhoça-SC, Brasil.

3.Fisioterapeuta, Doutor em Ciências da Saúde, Universidade do Sul de Santa Catarina, Palhoça-SC, Brasil. 4.Fisioterapeuta, Especialista em Fisioterapia Neurofuncional, Universidade do Sul de Santa Catarina, Palhoça-SC, Brasil.

5.Fisioterapeuta, Especialista em Fisioterapia Neurofuncional e docente do curso de Fisioterapia, Universidade do Sul de Santa Catarina, Palhoça-SC, Brasil.

\begin{abstract}
Resumo
Introdução. A atrofia muscular espinhal (AME) é uma doença neuromuscular degenerativa de caráter progressiva. Há diversas abordagens terapêuticas para que se minimize as progressões advindas da doença. O prognóstico favorável é altamente dependente tanto do diagnóstico precoce, quanto do início prematuro do tratamento medicamentoso e de todas as intervenções, dentre elas, a fisioterapia. A intervenção fisioterapêutica pode proporcionar maior sobrevida e com maior qualidade de vida aos pacientes. Objetivos. Analisar, por meio de uma revisão bibliográfica, quais foram as intervenções fisioterapêuticas aplicadas na atrofia muscular espinhal nos últimos 10 anos. Método. Revisão bibliográfica de artigos publicados nas bases de dados Biblioteca Virtual em Saúde (BVS), Scielo, Pubmed, LILACS, e Physiotherapy Evidence Database (PEDro). Resultados. Dos 162 artigos encontrados, apenas 6 artigos cumpriram todos os critérios de inclusão. Foram encontradas formas de avaliação e de recursos fisioterapêuticos utilizados para o tratamento da AME. Conclusões. Diante da pesquisa, verificou-se que as principais intervenções fisioterapêuticas utilizadas na AME nos últimos 10 anos foram exercícios em plataformas vibratórias, exercícios de fortalecimento com resistência, exercícios aquáticos e exercícios respiratórios.
\end{abstract}

Unitermos. AME; fisioterapia; doenças neuromusculares

\begin{abstract}
Introduction. Spinal muscular atrophy (SMA) is a progressive degenerative neuromuscular disease. It has several therapeutic approaches to minimize the progression of the disease. The favorable prognosis is highly dependent on the early diagnosis, from the beginning to the treatment with medications and even interventions, among them physiotherapy. Research has shown that physiotherapeutic intervention provides assistance to patient survival in a better quality of life. Objective. To measure, through a review of the literature, the physiotherapeutic interventions implemented in spinal muscular atrophy in the last ten years. Method. A literature review of articles published in the databases of the Virtual Health Library, Scielo, Pubmed, LILACS and Physical Therapy Evidence Database (PEDro). Results. Of the 162 articles identified, only six articles met the inclusion criteria. were found various forms of assessment and of physiotherapeutic resources for the treatment of SMA. Conclusions. the main physical therapy interventions for SMA in the last ten years were exercises on
\end{abstract}


vibratory platform, strengthening with resistance exercises, aquatic therapeutic exercises and breathing exercises were found.

Keywords. SMA; physical therapy; neuromuscular diseases

\begin{abstract}
Resumen
Introducción. Atrofia muscular espinal (AME) es una enfermedad neuromuscular degenerativa progresiva. Existen varios enfoques terapéuticos para minimizar la progresión de la enfermedad. El pronóstico favorable depende en gran medida del diagnóstico precoz, del inicio prematuro del tratamiento farmacológico y de todas las intervenciones, incluida la fisioterapia. La intervención de fisioterapia puede proporcionar una supervivencia más larga y una mejor calidad de vida para los pacientes. Objetivos. Analizar, mediante una revisión bibliográfica, cuáles fueron las intervenciones de fisioterapia aplicadas en la atrofia muscular espinal en los últimos 10 años. Método. revisión bibliográfica de los artículos publicados en las bases de datos de la Biblioteca Virtual en Salud (BVS), Scielo, Pubmed, LILACS y Physiotherapy Evidence Database (PEDro). Resultados: De los 162 artículos encontrados, solo 6 artículos cumplieron con todos los criterios de inclusión. Se encontraron formas de evaluación y recursos de fisioterapia utilizados para el tratamiento de la enfermedad. Conclusión. En vista de la investigación, se encontró que las principales intervenciones de fisioterapia utilizadas en la atrofia muscular espinal en los últimos 10 años fueron ejercicios en plataformas vibratorias, ejercicios de fortalecimiento con resistencia, ejercicios acuáticos y ejercicios de respiración.
\end{abstract}

Palabras clave. atrofia muscular espinal; fisioterapia; enfermedades neuromusculares

Trabalho realizado na Universidade do Sul de Santa Catarina, Florianópolis-SC, Brasil.

\title{
INTRODUÇÃO
}

A atrofia muscular espinhal (AME) é uma doença neuromuscular degenerativa, progressiva e debilitante com incidência de 1 em cada 10.000 nascidos vivos. Na AME há uma mutação ou deleção do gene de sobrevivência de neurônio motor 1 (SMN - Survival Motor Neuron). A doença é clinicamente dividida em cinco subgrupos de acordo com a gravidade e idade do início dos sintomas, variando entre o tipo 0 , considerado a forma mais grave até o tipo IV, considerado a forma mais branda ${ }^{1,2}$.

O tipo 0 é descrito para neonatos que durante a gestação apresentaram fraqueza muscular e hipotonia grave 
com diminuição dos movimentos fetais. Durante os exames pré-natais os bebês podem apresentar arreflexia, defeitos do septo atrial, contraturas articulares e insuficiência respiratória.

O tipo I, também conhecida como síndrome de Werdnig-Hoffman, apresenta hipotonia, déficit motor, fraqueza muscular grave e generalizada associada à insuficiência respiratória. Ocorrem fasciculações, diminuições dos reflexos profundos, além do acometimento dos pares cranianos.

O tipo II pode apresentar insuficiência respiratória, terá capacidade de sentar e dificuldade para deambular sem auxílio, também haverá fraqueza muscular que afetará inicialmente os membros inferiores e a região proximal do tronco. A escoliose associada à fraqueza muscular intercostal pode causar doença pulmonar restritiva.

O tipo III, também conhecida como doença de Kugelberg-Welander, possuí atraso no desenvolvimento neuropsicomotor associado à fadiga muscular leve e fraqueza em região proximal do quadril, fazendo com que possa necessitar do uso de cadeira de rodas ou consiga até mesmo deambular. Não apresenta grandes chances de escolioses e apresenta pouca ou nenhuma fraqueza da musculatura respiratória.

Se o início dos sintomas for antes dos 3 anos de idade é denominado como AME tipo IIIa e após essa idade é classificado como AME tipo IIIb. A diferença entre ambas é a capacidade dos indivíduos da AME IIIa andar até os 20 anos 
de idade, enquanto na AME tipo IIIb permanecem com a habilidade de deambular durante toda a sua vida.

O tipo IV tem início dos sintomas com fraqueza muscular apenas na fase adulta, haverá comprometimento motor leve, não alterando a deambulação ${ }^{1-5}$.

Cerca de 95\% dos pacientes com AME tem a ausência do gene de sobrevivência de neurônio motor SMN1 por deleção ou conversão gênica de SMN1 para SMN2. Os 5\% restantes, têm uma mutação no gene SMN1 que tipicamente perturba a produção de proteína SMN totalmente funcional. Usa-se o genótipo de SMN1 para diagnosticar AME, onde são realizados estudos genéticos moleculares e análise quantitativa dos genes SMN, que verificará deleções e conversões do gene SMN1, além de eletromiografia que verificará se há acometimento do neurônio motor, de raízes nervosas, fibras musculares ou de nervos periféricos e a biópsia muscular verificará se há alteração histopatológica ${ }^{6,7}$.

Atualmente a AME não tem cura, porém um tratamento multiprofissional pode aumentar a sobrevida e proporcionar uma melhor qualidade de vida aos portadores. O tratamento medicamentoso, consiste em aumentar o nível de produção ou estabilizar a proteína $\mathrm{SMN}^{6,8,9}$.

Diferentes abordagens terapêuticas reduzem a progressão da AME. O prognóstico favorável é altamente dependente do diagnóstico precoce, início do tratamento medicamentoso e de todas demais intervenções. Diante disto aumentam-se a sobrevida e preserva-se a funcionalidade do indivíduo. Entre estas abordagens a intervenção 
fisioterapêutica auxilia na sobrevivência e na qualidade de vida dos pacientes, o que vem melhorando na última década devido a mudanças e atualização dos cuidados ${ }^{6,9}$.

No exercício profissional do fisioterapeuta existem desafios para a sobrevivência da criança com AME, a fim de reduzir ou evitar complicações musculoesqueléticas atuando na hipotonia, nas deformidades e reduzindo as chances de complicações respiratórias. A intervenção fisioterapêutica durante todo desenvolvimento das crianças com AME é primordial, levando em consideração que interações funcionais com os segmentos corporais e com o meio ambiente em que residem irão contribuir para que haja desenvolvimento durante seu crescimento ${ }^{10}$.

Assim, o objetivo deste estudo foi descrever por meio de uma revisão bibliográfica as intervenções fisioterapêuticas na atrofia muscular espinhal nos últimos 10 anos.

\section{MÉTODO}

O presente estudo caracterizou-se como uma revisão de literatura, que abrangeu a leitura, análise e interpretação de artigos publicados na íntegra, em periódicos científicos disponibilizados na internet. As bases de dados utilizadas foram Biblioteca Virtual em Saúde (BVS), Scielo, Pubmed, LILACS, e Physiotherapy Evidence Database (PEDro). Para realizar a busca nas referidas bases foram utilizados os seguintes descritores e termos livres: "atrofia muscular espinhal", "AME" combinadas com "fisioterapia". Os 
respectivos termos em inglês "Muscular Atrophy Spinal" e "physiotherapy" também foram utilizados na busca.

Foram incluídos artigos publicados entre janeiro de 2009 a abril de 2019, disponíveis online na íntegra, nos idiomas português e inglês, que abordaram a atuação da fisioterapia na atrofia muscular espinhal. Foram adotados como critérios de exclusão estudos experimentais, questionários e revisão, e aqueles que não apresentaram e/ou detalharam a intervenção fisioterapêutica realizada.

A análise dos artigos foi realizada extraindo os seguintes dados: (a) referências; (b) objetivos; (c) tamanho amostral e classificação dos pacientes quanto ao tipo de AME; (d) descrição do tratamento fisioterapêutico; (e) resultados.

\section{RESULTADOS}

Dos 162 artigos encontrados, apenas 6 artigos cumpriram todos os critérios de inclusão. Destes 162 artigos encontrados, 141 foram excluídos inicialmente por não apresentarem as palavras chaves no título ou por não possuírem os critérios exigidos no resumo. Dos 21 estudos selecionados para serem lidos na íntegra, 15 foram excluídos por não se enquadrarem nos critérios de inclusão, como por exemplo, não apresentar a fisioterapia detalhada, manobras apenas para desmame ventilatório ou por não abordar a AME. As características dos estudos selecionados para análise nesta revisão encontram-se descritos na Tabela 1. 
Tabela 1. Características dos estudos encontrados.

\begin{tabular}{|c|c|c|c|}
\hline $\begin{array}{c}\text { AUTOR/ } \\
\text { REFERÊNCIA }\end{array}$ & TÍTULO & AMOSTRA & AVALIAÇÃO \\
\hline Stark $2018^{11}$ & $\begin{array}{l}\text { Programa de treinamento domiciliar } \\
\text { assistido por vibração para crianças } \\
\text { com atrofia muscular espinhal. }\end{array}$ & $\begin{array}{c}\mathrm{n}=38 \text { indivíduos } \\
\text { Tipo II }=28 \\
\text { Tipo III }=10\end{array}$ & $\begin{array}{c}\text { Gross Motor Function Measure } \\
\text { (GMFM-66); } \\
\text { Hammersmith Functional Motor } \\
\text { Scale (HFMS). }\end{array}$ \\
\hline Lewelt $2015^{12}$ & $\begin{array}{l}\text { Exercício de treinamento de força } \\
\text { com resistência em crianças com } \\
\text { atrofia muscular espinhal. }\end{array}$ & $\begin{array}{c}\mathrm{n}=9 \text { indivíduos } \\
\text { Tipo II }=6 \\
\text { Tipo III }=3\end{array}$ & $\begin{array}{c}\text { Dinamometria; } \\
\text { Escala de faces de Wong- } \\
\text { Baker; } \\
\text { Teste muscular manual; } \\
\text { Hammersmith Functional Motor } \\
\text { Scale (HFMS). }\end{array}$ \\
\hline Marques $2014^{13}$ & $\begin{array}{l}\text { Empilhamento de ar: efeitos sobre a } \\
\text { função pulmonar em pacientes com } \\
\text { atrofia muscular espinhal e em } \\
\text { pacientes com distrofia muscular } \\
\text { congênita (DMC). }\end{array}$ & $\begin{array}{c}\mathrm{n}=18 \text { indivíduos } \\
10 \text { indivíduos com DMC. } \\
8 \text { indivíduos com AME. } \\
\text { Tipo II }=4 \\
\text { Tipo III }=4 \\
\end{array}$ & $\begin{array}{l}\text { Pneumotacografia; } \\
\text { Espirometria. }\end{array}$ \\
\hline Vry $2014^{14}$ & $\begin{array}{l}\text { Treinamento vibratório de corpo } \\
\text { inteiro em crianças com distrofia } \\
\text { muscular de Duchenne (DMD) e } \\
\text { atrofia muscular espinhal. }\end{array}$ & $\begin{array}{c}\mathrm{n}=22 \text { indivíduos } \\
14 \text { indivíduos com DMD } \\
8 \text { indivíduos com AME }\end{array}$ & $\begin{array}{c}\text { Exame clínico geral e } \\
\text { neurológico; } \\
\text { Exames laboratoriais; } \\
\text { Teste de caminhada de } 6 \\
\text { minutos; } \\
\text { Caminhada de } 10 \text { m, } \\
\text { Escala modificada do Medical } \\
\text { Research Council; } \\
\text { Dinamometria de mão; } \\
\text { Goniometria. }\end{array}$ \\
\hline Salem $2010^{15}$ & $\begin{array}{l}\text { Terapia aquática para uma criança } \\
\text { com atrofia muscular espinhal tipo } \\
\text { III: relato de caso. }\end{array}$ & $\begin{array}{c}\mathrm{n}=1 \text { indivíduo } \\
\text { Tipo III }\end{array}$ & $\begin{array}{c}\text { Gross Motor Function Measure } \\
\text { (GMFM-88); } \\
\text { Peabody Developmental Motor } \\
\text { Scales-Second Edition (PDMS- } \\
\text { 2), } \\
\text { Sistema GAITRite. }\end{array}$ \\
\hline $\begin{array}{l}\text { Nygren-Bonnier } \\
2009^{16}\end{array}$ & $\begin{array}{l}\text { Respiração glossofaríngea para } \\
\text { insuflação pulmonar em crianças com } \\
\text { atrofia muscular espinhal tipo II. }\end{array}$ & $\begin{array}{c}\mathrm{n}=11 \text { indivíduos } \\
\text { Tipo II }\end{array}$ & $\begin{array}{c}\text { Espirometria estática e } \\
\text { dinâmica; antropometria; Borg } \\
\text { Scale CR-10. }\end{array}$ \\
\hline
\end{tabular}

Dos seis artigos selecionados, dois estudos abordaram a terapia com vibração, um estudo relatou exercícios de fortalecimento muscular, outros dois estudos descreveram a realização de exercícios respiratórios e um estudo abordou terapia aquática. Nota-se que os estudos encontrados utilizaram instrumentos diversificados para a realização da avaliação fisioterapêutica (Tabela 2). 
Tabela 2. Resultados dos estudos encontrados.

\begin{tabular}{|c|c|c|c|}
\hline $\begin{array}{c}\text { AUTOR/ } \\
\text { REFERÊNCIA } \\
\end{array}$ & INTERVENÇÃO & $\begin{array}{l}\text { DURAÇÃO/ } \\
\text { FREQUENCIA }\end{array}$ & PRINCIPAIS RESULTADOS \\
\hline Stark $2018^{11}$ & $\begin{array}{l}\text { Exercícios na plataforma vibratória } \\
\text { nas posições em pé, sentada e de } 4 \\
\text { apoios. } \\
\text { Treinamento de resistência funcional, } \\
\text { fisioterapia aquática e treinamento } \\
\text { em esteira. }\end{array}$ & $\begin{array}{l}\text { No ambiente hospitalar a } \\
\text { fisioterapia consistiu em } \\
5 \text { horas. Dentre eles a } \\
\text { vibração: } 3 x \text { ao dia por } 3 \\
\text { minutos. Os outros } \\
\text { componentes da } \\
\text { intervenção foram } \\
\text { aplicados } 2 \text { a } 3 x \text { por } \\
\text { semana durante a } \\
\text { internação. } \\
\text { Vibração no ambiente } \\
\text { domiciliar: } 10 x \text { por } \\
\text { semana por } 3 \text { minutos. }\end{array}$ & $\begin{array}{l}\text { Não houve melhora na função } \\
\text { motora grossa }(p=0,124) ; \\
\text { Melhora na mobilidade } \\
\text { funcional após } 6 \text { meses de } \\
\text { treinamento }(p=0,007) .\end{array}$ \\
\hline Lewelt $2015^{12}$ & $\begin{array}{c}\text { Exercícios resistidos de flexores e } \\
\text { extensores do ombro, flexores e } \\
\text { extensores do cotovelo, flexores e } \\
\text { extensores do quadril, e extensores } \\
\text { do joelho. }\end{array}$ & $\begin{array}{l}\text { A intervenção foi de } 12 \\
\text { semanas, sessões com } \\
\text { duraçãa de } 60 \text { minutos. } \\
\text { Os participantes } \\
\text { exercitaram-se } 3 \times \text { por } \\
\text { semana, realizando } 2 \\
\text { séries de } 15 \text { repetições. }\end{array}$ & $\begin{array}{l}\text { Aumento de força }(p=0,01) \\
\text { sem alteração do esforço } \\
\text { realizado }(p=0,76) \\
\text { Aumento da função motora } \\
(p=0,04) ;\end{array}$ \\
\hline Marques $2014^{13}$ & Manobras de empilhamento de ar. & $\begin{array}{c}\text { Foram submetidos a } \\
\text { manobras diárias de } \\
\text { empilhamento de ar em } \\
\text { casa com ressuscitadores } \\
\text { manuais por } 4 \text { a } 6 \\
\text { meses. }\end{array}$ & $\begin{array}{c}\text { Houve melhorias no pico de } \\
\text { fluxo de tosse assistida e não } \\
\text { assistida } \\
(p<0,0001) \text {. } \\
\text { Nos pacientes sem escoliose, } \\
\text { houve aumento da capacidade } \\
\text { vital forçada }(p<0,05) \text {. } \\
\end{array}$ \\
\hline Vry $2014^{14}$ & $\begin{array}{c}\text { Exercício físico na plataforma } \\
\text { vibratória. }\end{array}$ & $\begin{array}{l}\text { Duração de } 3 \text { minutos, } \\
2 x \text { por dia com intervalo } \\
\text { mínimo de } 4 \text { horas entre } \\
\text { as duas sessões, } 5 x \text { na } \\
\text { semana; } \\
\text { por } 8 \text { semanas. }\end{array}$ & $\begin{array}{l}\text { Aumento de força e da função } \\
\text { muscular; } \\
\text { Melhora no teste de caminhada } \\
\text { de } 6 \text { minutos }(p<0,01) ; \\
\text { o grau angular de dorsiflexão } \\
\text { do tornozelo melhorou } \\
\text { ligeiramente. }\end{array}$ \\
\hline Salem $2010^{15}$ & $\begin{array}{l}\text { Treino de marcha; } \\
\text { treinamento de resistência e } \\
\text { equilíbrio; } \\
\text { atividades motoras; fortalecimento e } \\
\text { educação familiar; } \\
\text { aquecimento e exercícios aquáticos. }\end{array}$ & $\begin{array}{c}\text { Fisioterapia aquática } 2 x \\
\text { por semana, duração de } \\
45 \text { minutos por } 14 \\
\text { semanas. } \\
\text { Continuou o tratamento } \\
\text { domiciliar já existente } \\
\text { com fisioterapia } 4 x \text { por } \\
\text { semana e terapia } \\
\text { ocupacional } 2 x \text { por } \\
\text { semana. }\end{array}$ & $\begin{array}{l}\text { Melhora da força muscular em } \\
\text { MMII; } \\
\text { Melhora dos movimentos } \\
\text { pélvicos durante a caminhada; } \\
\text { redução da marcha em } \\
\text { tesoura; Aumento da função } \\
\text { motora grossa e fina. }\end{array}$ \\
\hline $\begin{array}{l}\text { Nygren-Bonnier } \\
2009^{16}\end{array}$ & $\begin{array}{l}\text { Vídeo instrutivo, informações por } \\
\text { escrito, treinamento e prática da } \\
\text { técnica de respiração glossofaríngea. }\end{array}$ & $\begin{array}{l}\text { 4x por vezes por semana } \\
\text { por } 8 \text { semanas. Não foi } \\
\text { informado a duração das } \\
\text { sessões. }\end{array}$ & $\begin{array}{l}\text { Efeitos positivos na capacidade } \\
\text { vital inspiratória, pico de fluxo } \\
\text { expiratório e expansão } \\
\text { torácica. }\end{array}$ \\
\hline
\end{tabular}

DISCUSSÃO

A AME resulta em fraqueza muscular progressiva e requer um tratamento multiprofissional para uma melhor qualidade de vida dos indivíduos, sendo a fisioterapia um dos 
principais tratamentos que buscam minimizar as progressões advindas da doença ${ }^{17}$. A AME é descrita pela diminuição da força muscular e da função motora, onde cerca de $100 \%$ dos pacientes com AME tipo I e 73\% dos pacientes com AME tipo II precisavam de assistência no autocuidado; cerca de 60\% dos pacientes do tipo III obtiveram independência funcional ${ }^{18}$.

No quesito mobilidade $90 \%$ dos pacientes com AME tipo I, II, IIIa e 63\% do tipo IIIb precisam de assistência para sua mobilidade, sendo constatado que a força e a função muscular possuem degeneração progressiva. A fraqueza generalizada grave é vista em pacientes com AME tipo 0, I e II e a fraqueza muscular proximal moderada em paciente com AME tipo III e IV5,18,19. Pacientes com AME tipo II e III possuem perda de $5 \%$ da força extensora do joelho e $20 \%$ para os flexores do joelho e cotovelo comparando com a população de idade e sexo iguais ${ }^{20}$, demonstrando desta forma a fraqueza progressiva adquira pelos portadores de AME.

A fadiga muscular é uma queixa comum em pacientes com doenças neurológicas, dentre elas, a AME, prejudicando a qualidade de vida desses indivíduos. Avaliando a gravidade da fadiga muscular em pacientes com doenças neuromusculares, inclusive em indivíduos com AME, foi investigado se a fadiga é atribuída ao bloqueio de condução dependente de atividade, através de eletromiografia de fibra única com uma hiperpolarização axonal. Os resultados mostraram que a porcentagem de pacientes com fadiga foi 
maior no grupo com AME e no grupo com atrofia muscular bulbo espinhal ${ }^{21}$.

Considerando que a AME é uma doença que apresenta comprometimento de força e resistência muscular, determinadas modalidades de treinamento podem ser promissoras para o tratamento da degeneração causada pela doença. Foi avaliado o resultado de exercícios de resistência em crianças com AME submetidas a um plano de exercícios resistido progressivo com o objetivo de aumentar e melhorar a função motora ${ }^{12}$. A intervenção teve duração de 12 semanas consecutivas compreendendo um programa domiciliar supervisionado por um fisioterapeuta. Como resultados, houve aumento do peso levantado pelos participantes e o nível de esforço percebido permaneceu inalterado. Nos membros superiores, através do teste muscular manual houve aumento da força. Esta mudança foi atribuída ao aumento da força em flexores e extensores de ombro, e flexores de cotovelo. Os escores HFMS aumentaram, o que demostra que a melhora da força trouxe resultados funcionais ao paciente ${ }^{12}$.

Estudo aponta que miopatias inflamatórias idiopáticas crônicas, possuem redução de força e da resistência muscular, bem como fadiga e mialgia ${ }^{22}$. Diante disto, o seu estudo demostrou que o exercício físico pode ser uma forma de neutralizar vários dos mecanismos que possuem um efeito negativo na função muscular em indivíduos com comprometimento degenerativo. Com isso, avaliaram o potencial benéfico de um programa de treinamento muscular 
resistivo intensivo em pacientes com polimiosite crônica e dermatomiosite. Em sete semanas de treinamento de resistência muscular, houve diferença na força, deltoide direito, gastrocnêmios e quadríceps esquerdo. Os músculos deltoide esquerdo, quadríceps direito e músculos abdominais tiveram um ganho de força considerável. Houve melhora da resistência muscular na flexão de ombro ${ }^{22}$. Diante desses resultados, confirma-se que exercícios resistidos promovem ganho funcional durante 0 tratamento de doenças degenerativas, como a AME. Tratamento este, que contribuirá para o fortalecimento, manutenção e funcionalidade destes indivíduos.

Outra modalidade terapêutica que tem sido utilizada para incremento da força muscular é o treinamento em plataforma vibratória. Os movimentos oscilatórios realizados pela vibração de corpo inteiro são de natureza mecânica. Tais movimentos estimulam fisiologicamente o corpo humano de forma que haja a busca da estabilização postural, assim como também há estimulação dos fusos musculares juntamente com a ação dos motoneurônios que agem na ativação do reflexo de estiramento ${ }^{23,24}$. Na AME há degeneração do motoneurônio alfa e consequentemente fraqueza muscular proximal progressiva e atrofia dos músculos esqueléticos ${ }^{25}$. Nos músculos dos portadores de AME não há o recebimento de estímulos eferente suficiente, sendo uma das causas da atrofia.

A plataforma vibratória é benéfica no tratamento em pacientes com AME atuando diretamente nos músculos 
atrofiados provocando reflexos musculares, fortalecendo e prevenindo efeitos a longo prazo causados pela hipomobilidade ${ }^{11,14}$. Verificou-se o efeito da vibração em 22 indivíduos, sendo oito com $\mathrm{AME}^{14}$. A frequência foi gradativamente aumentada, de $10 \mathrm{~Hz}$ no primeiro dia até $24 \mathrm{~Hz}$ na última semana. Foram utilizados testes funcionais, além de exames de força muscular através da escala modificada Medical Research Council (MRC) e grau angular através do goniômetro. Ao final do estudo não houve dados consideráveis, apenas no teste de caminhada de seis minutos, porém foi perceptível a melhora, mesmo que mínima em grande parte das avaliações realizadas, principalmente nos testes funcionais, na força muscular e grau angular, visto que por ser uma doença progressiva não obter resultados retrógrados já é satisfatório, principalmente por constar que não há sequelas advindas do tratamento ${ }^{14}$.

Verificou-se o efeito da vibração em 38 indivíduos com AME tipo II e III, a intervenção alternou períodos de internação hospitalar com tratamento intensivo, associado a períodos de tratamento domiciliar ${ }^{11}$. Foram avaliadas crianças através das escalas GMFM-66 e HFMS antes da intervenção, seis e 12 meses após. Os resultados mostraram que as crianças avaliadas através da GMFM-66 não apresentaram aumento da função motora grossa após 12 meses e através da HFMS apresentaram aumento na mobilidade funcional ${ }^{11}$. Diante destes resultados, é visto que, diante das diversas abordagens atuais encontradas na literatura e na prática clínica, a vibração é um meio de 
tratamento relevante, que atuará tanto no fortalecimento muscular quanto na busca da mobilidade funcional dos pacientes.

O efeito da plataforma vibratória com frequência $18 \mathrm{~Hz}$, foi observado em dois pacientes com doença de Pompe, onde foi utilizada a vibração em membros inferiores (postura em pé) e nos membros superiores (mãos na plataforma, cotovelo em extensão, ajoelhados no chão) ${ }^{26}$. A duração da vibração foi de 30 segundos em cada região, sendo realizado 3 vezes por semana durante 2 anos. Ao final dos 24 meses, concluiu-se que o tratamento com vibração pode ser utilizado com segurança em pacientes com doença de Pompe e que houve um aumento moderado na força muscular destes pacientes ${ }^{26}$.

Além da fraqueza e fadiga muscular, pacientes com AME também apresentam comprometimento respiratório, assim como em outras doenças neuromusculares ${ }^{27}$. Em pacientes com doença neuromuscular, a exigência de consumo de oxigênio é de duas a três vezes maior. Com isso, a utilização da musculatura acessória respiratória possui uma carga alta de trabalho, para manter um nível reduzido de função e homeostase gasosa. Por este motivo, sugere-se que técnicas de fisioterapia respiratória possam auxiliar no manejo de pacientes com AME. Foi avaliado o efeito da respiração glossofaríngea na função pulmonar e na expansão torácica de crianças com AME do tipo $\mathrm{II}^{16}$. Crianças que conseguiram executar esta técnica adequadamente, obtiveram efeitos positivos do pico de fluxo expiratório (PFE) 
na qual houve uma mudança média de 116litros/minuto. Na capacidade vital inspiratória (CVI) observou-se uma mudança média de 0,131 . Na expansão torácica observou-se um aumento médio de $1,79 \mathrm{~cm}$. Concluíram então que, uma vez aprendida a técnica, esta pode ser realizada em casa com auxílio dos pais para melhorar a CVI e PFE de indivíduos que possuem AME tipo $\mathrm{II}^{16}$.

Um estudo com o objetivo comparar os efeitos da respiração glossofaríngea com empilhamento de ar em 14 pacientes com doenças neuromusculares, foi observado que a mediana da capacidade vital no empilhamento de ar melhorou e a respiração glossofaríngea aumentou, com diferença de $290 \mathrm{ml}$ entre as duas técnicas ${ }^{28}$. Concluindo que ambas são eficazes no tratamento e ressaltaram que os resultados foram mais positivos no empilhamento de ar devido à dificuldade no aprendizado da técnica da respiração glossofaríngea.

Outro estudo buscou determinar os efeitos das manobras diárias de empilhamento de ar sobre a função pulmonar em pacientes com AME II e III e distrofia muscular congênita, além de associar as deformidades da coluna com os efeitos desta técnica ${ }^{13}$. A técnica foi realizada com ressuscitador manual e máscara facial acoplada à face do paciente. Após o uso das manobras de empilhamento de ar no domicílio, houve uma melhora no pico de fluxo de tosse assistido e não assistido. Nos pacientes sem escoliose, houve também aumento na capacidade vital forçada. No grupo de pacientes sem escoliose, o ganho no pico de fluxo de tosse 
assistido e não assistido foi superior ao do grupo com escoliose. Apesar das limitações do estudo devido ao pequeno tamanho de amostra, a dificuldade em realizar as manobras de empilhamento de ar e a aplicação não ser supervisionada no domicílio, por exemplo, os pesquisadores concluem que estas manobras devem ser prescritas para pacientes com doenças neuromusculares a fim de aumentar o pico de fluxo de tosse ${ }^{13}$.

Em pacientes com doenças neuromusculares além da insuficiência da musculatura inspiratória, a perda progressiva da capacidade vital e fraqueza dos músculos expiratórios causam insuficiência da tosse, ocasionando infecções recorrentes e atelectasias. Foi verificado o uso de insuflação/exsuflação mecânica (MI-E) em 21 pacientes com doenças neuromusculares que possuíam tosse ineficiente ${ }^{29}$. Os pacientes foram instruídos a usar a MI-E duas vezes ao dia durante 10 minutos a fim de evitar o declínio da capacidade vital. No primeiro ano, após o uso regular MI-E houve um aumento em $28 \%$ da capacidade vital e no segundo ano o aumento permaneceu estável. O uso da MI-E pode melhorar a mobilidade do tórax rígido e ajudar na remoção de secreções retidas. Diante disto, concluíram que a MI-E é eficaz na melhora de microatelectasias e a complacência pulmonar, fato na qual se reduz a hospitalização e a mortalidade 29 .

Distúrbios como fraqueza muscular, fadiga e complicações respiratórias ocasionam disfunções marcantes da função motora de portadores de AME. Sendo assim, a 
fisioterapia aquática proporciona o bem-estar do indivíduo com e sem lesão neurológica ou com alterações musculares, cardiorrespiratórias ou funcionais. Os exercícios aquáticos estimulam o ajuste postural para a execução das habilidades motoras e reações de equilíbrio, além de promover benefícios terapêuticos e fisiológicos, como a diminuição da dor, consequentemente possibilita a maior mobilidade funcional dos indivíduos. Tais ganhos ocasionados pela fisioterapia aquática são conquistados devido as propriedades físicas e a temperatura da água ${ }^{30}$.

Em um estudo com o objetivo de avaliar as mudanças da função motora após um programa de terapia aquática em uma criança de três anos com AME tipo III $^{15}$, foi realizada fisioterapia aquática, duas vezes por semana com duração de 45 minutos, por 14 semanas com temperatura da água em $33^{\circ} \mathrm{C}$. Como resultado da intervenção a pontuação GMFM total melhorou em $11 \%$, na dimensão em pé melhorou $28 \%$ e na dimensão caminhada, corrida e salto aumentou $18 \%$. Porém a fisioterapia aquática não foi a intervenção exclusiva, sendo associada a fisioterapia no solo e a terapia ocupacional ${ }^{15}$.

Em um estudo de caso, com o objetivo de verificar a interferência da fisioterapia aquática na agilidade de uma criança com distrofia muscular de Duchenne não deambuladora, com 12 anos de idade, do sexo masculino ${ }^{31}$. Realizou 10 sessões de fisioterapia aquática, em uma piscina coberta e aquecida com temperatura da água de $32{ }^{\circ} \mathrm{C}$, com duração de 60 minutos cada sessão. A intervenção foi 
constituída de um protocolo de exercícios com enfoque na agilidade na cadeira de rodas. Como resultados, houve melhoras na saturação de oxigênio (de 93\% para 96\%), e pressão inspiratória (de $-40,00$ para -45). Diante da progressão natural da doença, ao reavaliar a agilidade do indivíduo, no teste de zigue-zague houve uma queda de 125 s para 100s na execução dele. Com esses resultados, houve uma mudança quantitativa na agilidade do paciente avaliado, evidenciando uma diminuição no tempo de deslocamento na cadeira de rodas e melhorando alguns dos padrões respiratórios do indivíduo ${ }^{31}$ o que vai ao encontro dos resultados obtidos na $\mathrm{AME}^{15}$.

$\mathrm{Na} A M E$, especificamente parte dos resultados que se espera no tratamento na água advém dos efeitos térmicos sobre o tecido muscular, principalmente nos casos de encurtamento e outra parte se dá por questões mecânicas como alívio do peso corporal e sobre a mobilidade ${ }^{32}$.

Diante de todos os meios buscados a fim de trazer uma melhor qualidade de vida aos pacientes com AME, em dezembro de 2016 foi registrado o medicamento Nusinersena comercializado como Spinraza da empresa Biogen, aprovado como rótulo amplo, disponível para todas as idades e para todos os tipos da doença. Foi realizado estudo onde foram selecionadas 126 crianças, entre 2 e 12 anos, onde 84 foram randomizadas para o grupo nusinersena, ou seja, receberam $12 \mathrm{mg}$ da medicação e 42 no grupo controle, isto é, procedimento simulado ${ }^{33}$. Os resultados obtidos mostraram que $57 \%$ das crianças no 
grupo nusinersena tiveram um aumento de pelo menos três pontos, considerado clinicamente significativo, na escala HFMS e no grupo controle apenas $26 \%$ alcançaram esta pontuação. Foi observado que os resultados mais positivos foram em crianças menores e naquelas que obtiveram o tratamento logo após o início dos sintomas. No grupo controle, observou-se uma melhora na pontuação média até o sexto mês, na qual a diferença do avanço entre os grupos nusinersen e o controle tornaram-se mais aparente. Os pesquisadores constataram que a melhora inicial observada no grupo controle nos primeiros meses de tratamento se deu provavelmente pela combinação do efeito placebo, da curva de aprendizado e dos ganhos iniciais de desenvolvimento nas crianças mais novas. Estes resultados positivos devem levar em consideração a necessidade imediata de triagem neonatal, a fim de iniciar o tratamento no tempo ideal 33-35.

Diante da pesquisa realizada para este estudo identificou-se como limitações o número escasso de artigos que exploraram a fisioterapia na $A M E$ e que informassem quais intervenções fisioterapêuticas foram realizadas no tratamento. Assim como, não foi o propósito do estudo, cabe ainda relatar que não foi encontrado estudos que associassem a fisioterapia juntamente com o tratamento medicamentoso. Apesar disso, os estudos encontrados obtiveram resultados positivos para evitar a progressão da doença, fato este totalmente importante para uma melhor qualidade de vida dos pacientes. 
Os estudos encontrados nesta pesquisa fortalecem a orientação para prescrição e programação terapêutica a partir dos instrumentos de avaliação identificados, das rotinas e resultados obtidos. Identificou-se ainda a utilização da escala própria de avaliação para AME, que apesar de ter sido traduzida e adaptada para o português de acordo com a cultura brasileira ${ }^{36}$, ainda precisa ser validada e utilizada como uma rotina entres os profissionais no Brasil. Todos os estudos também descreveram o período e a intervenção utilizada no protocolo, informações estas pertinentes a todos fisioterapeutas que procuram o melhor tratamento para seus pacientes.

\section{CONCLUSÃO}

Sendo assim, as principais intervenções encontradas para o tratamento fisioterapêutico da AME nos últimos 10 anos foram exercícios em plataformas vibratórias em diversas posturas, sendo ou não associados a exercícios funcionais em solo, exercícios resistidos, manobras de empilhamento de ar, exercícios aquáticos e exercícios respiratórios.

Os resultados encontrados neste estudo apontam para a necessidade de mais estudos com ensaios clínicos e maior número de indivíduos para "desfechos" mais fidedigno. Estudos que tracem protocolos e realizem estudos com fisioterapia motora e respiratória associadas, assim como a fisioterapia associada à medicação. 


\section{REFERÊNCIAS}

1.Parente V, Corti S. Advances in spinal muscular atrophy therapeutics. Ther Adv Neurol Disord 2018;11:1-13. https://doi.org/10.1177/1756285618754501

2.Faravelli I, Nizzardo M, Comi GP, Corti S. Spinal muscular atrophy recent therapeutic advances for an old challenge. Nat Ver Neurol 2015; 11:351-9. https://doi.org/10.1038/nrneurol.2015.77

3.Kolb SJ, Kissel JT. Spinal Muscular Atrophy. Neurol Clin 2015;33:831-46. https://doi.org/10.1016/j.ncl.2015.07.004

4.Finkel RS, McDermott MP, Kaufmann P, Darras BT, Chung WK, Sproule DM, et al. Observational study of spinal muscular atrophy type I and implications for clinical trials. Neurology 2014;83:810-7. https://doi.org/10.1212/WNL.0000000000000741

5.Ministério da Saúde (endereço na internet). Parecer Referencial n. 00016/2017/CONJURMS/CGU/AGU.

Manifestação Jurídica referencial sobre o medicamento Spinrazaß

Nusinersen (acessado em 2020). Disponível em: http://portalarquivos.saude.gov.br/images/pdf/2017/maio/30/00001

6-2017--Spinraza-Nusinersen.pdf

6. Ruhno C, McGovern VL, Avenarius MR, Snyder PJ, Prior TW, Nery FC, et al. Complete sequencing of the SMN2 gene in SMA patients detects SMN gene deletion junctions and variants in SMN2 that modify the SMA phenotype. Hum Genet 2019;138:241-56.

https://doi.org/10.1007/s00439-019-01983-0

7.Cao Y-Y, Zhang W-H, Qu Y-J, Bai J-L, Jin Y-W, Wang $\mathrm{H}$; et al. Diagnosis of Spinal Muscular Atrophy: A Simple Method for Quantifying the Relative Amount of Survival Motor Neuron Gene 1/2 Using Sanger DNA Sequencing. Chin Med J 2018;131:2921-9.

https://doi.org/10.4103/0366-6999.247198

8. Riessland M. Is there hope for spinal muscular atrophy synthetic pharmacotherapy? Expert Opin Pharmacother 2019;20:1-4. https://doi.org/10.1080/14656566.2019.1595585

9.Pechmann A, König K, Bernert G, Schachtrup K, Schara U, Schorling $\mathrm{D}$, et al. SMArtCARE - A platform to collect real-life outcome data of patients with spinal muscular atrophy. Orphanet J Rare Dis 2019;14:18. https://doi.org/10.1186/s13023-019-0998-4

10.Kades MGP, Aquino MES, Laurindo Souza LP. Síndrome de WerdnigHoffman: Aspectos Patológicos e os Saberes da Enfermagem. Recien 2019;7:40-7.

https://doi.org/10.24276/rrecien2358-

3088.2017.7.20.40-47

11.Stark C, Duran I, Cirak S, Hamacher S, Hoyer-Kuhn H-K, Semler $\mathrm{O}$, et al. Vibration-Assisted Home Training Program for Children With Spinal Muscular Atrophy. Child Neurol Open 2018;5:1-9. https://doi.org/10.1177/2329048X18780477

12. Lewelt A, Krosschell KJ, Stoddard GJ, Weng C, Xue M, Marcus $\mathrm{RL}$, et al. Resistance strength training exercise in children with spinal muscular atrophy. Muscle Nerve 2015;52:559-67.

https://doi.org/10.1002/mus.24568 
13. Marques TBC, Neves JC, Portes LA, Salge JM, Zanoteli E, Reed UC. Air stacking: effects on pulmonary function in patients with spinal muscular atrophy and in patients with congenital muscular dystrophy. J Bras Pneumol 2014;40:528-34. https://doi.org/10.1590/s180637132014000500009

14.Vry J, Schubert IJ, Semler O, Haug V, Schonau E, Kirschner J. Whole-body vibration training in children with Duchenne muscular dystrophy and spinal muscular atrophy. Eur J Paediatr Neurol 2014;18:140-9. https://doi.org/10.1016/j.ejpn.2013.09.005

15.Salem Y, Gropack SJ. Aquatic Therapy for a Child with Type III Spinal Muscular Atrophy: A Case Report. Acta Fisiotr 2010;23:102-6. https://doi.org/10.3109/01942638.2010.493097

16.Nygren-Bonnier M, Markström A, Lindholm P, Mattsson E, Klefbeck B. Glossopharyngeal pistoning for lung insufflation in children with spinal muscular atrophy type II. Acta Paediatr 2009;98:1324-8. https://doi.org/10.1111/j.1651-2227.2009.01343.x

17.Koul R, Al-Futaisi A, Al-Thihli K, Bruwer Z, Scott P. Segmental Spinal Muscular Atrophy Localised to the Lower Limbs: First case from Oman. Sultan Qaboos Univ Med J 2017; e355-7.

https://doi.org/10.18295/squmj.2017.17.03.018

18.Chung $\mathrm{BH}$, Wong VC, Ip P. Spinal muscular atrophy: survival pattern and functional status. Pediatrics 2004;114:e548-53.

https://doi.org/10.1542/peds.2004-0668

19.Febrer A, Rodriguez N, Alias L, Tizzano E. Measurement of muscle strength with a handheld dynamometer in patients with chronic spinal muscular atrophy. J Rehabil Med 2010;42:228-31.

https://doi.org/10.2340/16501977-0507

20.Merlini L, Bertini E, Minetti C, Mongini T, Morandi L, Angelini C, et al. Motor function-muscle strength relationship in spinal muscular atrophy. Muscle Nerve 2004;29:548-52.

https://doi.org/10.1002/mus.20018

21. Noto Y-i, Misawa S, Mori M, Kawaguchi N, Kanai K, Shibuya K, et al. Prominent fatigue in spinal muscular atrophy and spinal and bulbar muscular atrophy: evidence of activity-dependent conduction block. Clin Neurophysiol 2013;124:1893-8.

https://doi.org/10.1016/j.clinph.2012.12.053

22.Alexanderson $H$, Dastmalchi $M$, Esbjörnsson-Liljedahl $M$, Opava $\mathrm{CH}$, Lundberg IE. Benefits of intensive resistence training in patients with chronic polyomyosits or dermatomyosits. Arthritis Rheum 2007;57:768-77. https://doi.org/10.1002/art.22780

23.Monazzam MR, Shoja E, Zakerian SA, Foroushani AR, Shoja $M$, Gharaee $M$, et al. Combined effect of whole-body vibration and ambient lighting on human discomfort, heart rate, and reaction time. Int Arch Occup Environ Health 2018;91:537-45.

https://doi.org/10.1007/s00420-018-1301-z

24.Alam MM, Khan AA, Farooq M. Effect of whole-body vibration on neuromuscular performance: A literature review. Work 2018;59:57183. https://doi.org/10.3233/WOR-182699 
25.Zanoteli E, Maximino JR, Reed UC, Chadi G. Spinal muscular atrophy: from animal model to clinical trial. Funct Neurol 2010;25:739.

26. Montagnese $\mathrm{F}$, Thiele $\mathrm{S}$, Wenninger $\mathrm{S}$, Schoser $\mathrm{B}$. Long-term wholebody vibration training in two late-onset Pompe disease patients. Neurol Sci 2016;37:1357-60. https://doi.org/10.1007/s10072-0162612-z

27.Fitzgerald DA, Doumit M, Abel F. Changing respiratory expectations with the new disease trajectory of nusinersen treated spinal muscular atrophy [SMA] type 1. Paediatr Respir Rev 2018;28:11-7.

https://doi.org/10.1016/j.prrv.2018.07.002

28.Torres-Castro R, Vilaró J, Vera-Uribe R, Vasconcello L, Puppo H. Acute Effects of Air Stacking Versus Glossopharyngeal Breathing in Patients with Neuromuscular Disease. BJMMR 2016,14:1-8. https://doi.org/10.9734/BJMMR/2016/23192

29.Stehling F, Bouikidis A, Schara U, Mellies U. Mechanical insufflation/exsufflation improves vital capacity in neuromuscular disorders. Chron Respir Dis 2015;12:31-5.

https://doi.org/10.1177/1479972314562209

30.Israel VL, Pardo MBL. Hydrotherapy: application of an Aquatic Functional Assessment Scale (AFAS) in aquatic motor skills learning. Am Int J Contemp Res 2014;4:42-52.

https://core.ac.uk/download/pdf/197151851.pdf

31.da Silva KM, Braga D, Hengels RC, Beas ARV, Rocco FM. The impact of aquatic therapy on the agility of a non-ambulatory patient with Duchenne Muscular Dystrophy. Acta Fisiátrica 2012;19:42-5. https://doi.org/10.5935/0104-7795.20120009 32.Bottura AP, Accacio LMP, Mazzitelli C. Effects of a kinesiotherapy and aquatic physiotherapy program onto the neuropsycomotor development. Fisioterap Pesq 2006;13:67-75. https://doi.org/10.1590/fpusp.v13i3.76315

33. Mercuri E, Darras BT, Chiriboga CA, Day JW, Campbell C, Connolly $A M$, et al. Nusinersen versus Sham Control in Later-Onset Spinal Muscular Atrophy. N Engl J Med 2018;378:625-35. https://doi.org/10.1056/NEJMoa1710504

34.Simoens S, Huys I. Market access of Spinraza (Nusinersen) for spinal muscular atrophy: intellectual property rights, pricing, value and coverage considerations. Gene Ther 2017;24:539-41.

https://doi.org/10.1038/gt.2017.79

35. Glascock J, Lenz M, Hobby K, Jarecki J. Cure SMA and our patient community celebrate the first approved drug for SMA. Gene Ther 2017;24:498-500. https://doi.org/10.1038/gt.2017.39

36. De Oliveira CM. Estudo da qualidade de vida em crianças e adolescentes com Atrofia Muscular Espinhal (Tese). Rio de Janeiro: Universidade Federal do Rio de Janeiro, 2010. https://www.livrosgratis.com.br/ler-livro-online-109876/estudo-daqualidade-de-vida-em-criancas-e-adolescentes-com-atrofia-muscularespinhal 